\section{Variation in the Human Genome}

(CiBa Foundation SYMposium)

\section{edited by Derek Chadwick \& Gail Cardew \\ John Wiley \& Sons \\ ISBN: $0.471-96152.3,1996, \$ 84.95$}

REVIEWED By RICHARD R. HUDSON

Department of Ecology and Evolution Biology University of California at Irvine Irvine, CA 92697

This book is a collection of 16 papers presented by a distinguished assemblage of scientists at a Ciba Foundation Symposium held in London in June 1995. Most of the papers review very briefly what is known about the genetics of a particular disease or disease-related phenotype. These include phenylketonuria, cystic fibrosis, fragile $\mathrm{X}$ syndrome, beta-thalassemia, susceptibility to tumor development, levels of plasma low density lipoprotein-cholesterol and blood pressure. The paper, "Control of beta-thalassemia by carrier screening, genetic counseling and prenatal diagnosis: the Sardinian experience" by A. Cao et al., tells the fascinating story of a medical program which has reduced the birth rate of thalassemia major from 1:250 to $1: 4000$, over the last twenty years. D. J. Weatherall provides a remarkably concise review of the diverse array of malaria related polymorphisms that appear to have arisen quite recently in human popula- tions. Other papers discuss the methods of identifying the genetic variation contributing to polygenic phenotypes. The three opening papers, which are the only ones that do not deal directly with human disease, discuss the population genetic theory of neutral variation, including discussion of coalescent methods, effects of demographic parameters on patterns of neutral variation, and the theory of microsatellite variation. In these papers some data are presented on mitochondrial variation in Amerindian populations and microsatellite variation in several other human populations. The book also includes a chapter on the (somatic) population genetics of variation among cells in a tumor within an individual, and a chapter on HLA variation around the world and its implications for human disease.

This is certainly not a how-to book. The methodologies of identifying the genetic basis of phenotypic variation are covered in a very superficial way. However, this book does provide useful short introductions to what is known about a variety of human genetic diseases. In particular, the variety of mutations that have been observed, their frequencies in different populations, as well as inferences about the mutational mechanisms and selective histories are discussed. More importantly, these papers and the discussions among the contributors which are included as addenda to each paper, provide insight about what is not known, and how difficult it may be to identify the genetic basis of common diseases. It seems clear that if many genes of small effect are involved, and if interactions between genes are as important as mean effects of individual genes, then progress may be very slow.

Ciba Foundation The discussions also make it clear that inferring the past selective history of variants which are now important in human disease may also be very difficult.

This book provides some sense of where we stand in our pursuit to elucidate the genetic basis of disease. Some remarkable strides have been taken. Determining the genetic basis for common human diseases will likely lead to an improved understanding of the causes, and may help in the design of efficient prevention programs targeted at precisely those individuals who can most benefit from them. Whereas it is clear that advances in the technologies for surveying human genetic variation will soon result in an enormous influx of information on variation across the entire human genome, it is not clear that the result will be rapid identification of the genes involved in common human diseases.

\section{The Perfect Baby:}

\section{A Pragmatic Approach to Genetics}

by Glenn McGee Rowman \& Littlefield Publishers, Inc. ISBN: 0-8476-8344-3 (paper), 1997, \$18.95

REVIEWED by BRUCE C. TRAPNELlL Genetic Therapy, Inc., Department of Virology 938 Clopper Road, Gaithersburg, MD 20878

For those interested in exploring the moral and social issues surrounding genetic engineering, the diagnosis and cure of genetic illnesses and the potential impact of applying these technologies to the human experience, The Perfect Baby provides a stimulating and informative introduction. The book is appropriately directed to a diverse audience and makes refreshing use of plain language to address relevant issues present in the choices we must now make in everyday life.

A picture of the landscape of genetic technology is first painted using broad strokes to illustrate the development of our understanding of heredity, the technologies of modern genomic research, our attempts to develop gene therapy, the difficult decisions attendant to prenatal genetic screening and the ageless questions about the social control of reproduction. Particularly vivid is the picture we are shown of eugenic practices at the turn of the century, in which "thousands of institutionalized Americans were sterilized during the 1930 's to prevent...a generation of imbeciles" and, under the far worse German laws, sterilization "was compulsory with respect to all people, institutionalized or not, who suffered from allegedly hereditary disabilities...or were grossly offensive." With these and similar passages, McGee reminds us of the importance and of our obligation to understand the ethics of genetic research and the relevance of its impact on our daily lives.

Following a provocative introduction which demands the reader's attention, McGee summarizes the optimistic opinions of notable and enthusiastic scien- 